\title{
Zugang für Behinderte zu modernen Kommunikationstechnologien
}

\author{
M. Egli
}

1 Dr. André Assimacopoulos, Newsletter Infosociety Nr. 56, Juli 2005.

2 Dr. iur. Caroline Hess Klein, Newsletter Infosociety Nr. 56, Juli 2005.

Korrespondenz: Michael Egli, lic. rer. publ. HSG eHealthCare.ch Trend Care AG Josef Müller-Weg 2

CH-6210 Sursee

Tel. 0419257689

Fax 0419257680

E-Mail: michael.egli@ehealthcare.ch

Internet: www.ehealthcare.ch
Mit der Verabschiedung der Internationalen Klassifikation der Funktionsfähigkeit, Behinderung und Gesundheit (ICF) im Jahr 2001 durch die Weltgesundheitsorganisation (WHO) fand ein weltweiter Paradigmenwechsel statt: Das Konzept der behinderten Person wurde durch dasjenige der Person in einer behindernden Situation ersetzt. Das ist eine fundamental neue Sichtweise. Beschrieben wird nicht mehr der Mangel, nicht mehr, was einer Person fehlt, nicht mehr deren Anomalien oder die Folgen einer Krankheit oder eines Unfalls. Die Aufmerksamkeit richtet sich vielmehr auf die Umgebung, in der sich die Person bewegt. Es ist die Unangepasstheit der Umgebung an die Funktionsweise dieses Menschen, die ihn behindert. In einem angepassten Umfeld gibt es keine Behinderung.

Wer stand noch nie vor einem Gerät mit einer kafkaesk anmutenden Bedienung, einer rätselhaften Gebrauchsanweisung und einer ebenso unverständlichen Anleitung! Es ist das ungeeignete und unangepasste Umfeld, das behindert und einschränkt. Die Entwicklung einer Technologie für Menschen mit einer besonderen Funktionsfähigkeit, das heisst mit einer aussergewöhnlichen Funktionsfähigkeit, ist ein Privileg. Sie zwingt den Entwickler, die Interaktion zwischen Mensch und Maschine in ihrer ganzen Komplexität zu analysieren, herauszufinden, in welcher Weise alle Sinne berücksichtigt werden müssen. Die Stiftung Zugang für alle (www. access-for-all.ch) leistet hier wertvolle Aufbauund Informationsarbeit [1].

\section{Entwicklungen Schweiz und weltweit}

Kommunikation bildet die Grundlage aller zwischenmenschlichen Beziehungen. In den letzten Jahren haben sich die Mittel dazu rasant entwickelt, vor allem Internet, Telefonie und Fernsehen. Dieser Prozess bietet eine enorme Chance, nicht zuletzt für die Demokratie, weil er Information und Diskussion erleichtert. Auch der Kauf von Gütern und die Nutzung verschiedener, früher schwer zugänglicher Dienstleistungen ist einfacher geworden. Für viele Behinderte bedeuten diese Kommunikationsmittel einen riesigen Fortschritt. So kann beispielsweise ein Tetraplegiker, der sich nur mit Mühe fortbewegt, ohne Probleme über das Internet einkaufen. Ebenso erhalten Blinde dank des Internets $\mathrm{Zu}$ gang zu Informationen - z.B. in den Printmedien -, die vorher nur mit Hilfe Dritter erreichbar waren. Bereits vor einigen Jahren fand in den angelsächsischen Ländern, vor allem in den USA, und in der Weltgesundheitsorganisation ein Wandel beim Ansatz gegenüber der Behinderung statt. Nicht die individuelle Behinderung wird als Ursache des Problems gesehen, sondern die mangelnde Anpassung der Gesellschaft. Anders ausgedrückt: Die Hör- oder Sehbehinderten können das Fernsehprogramm nicht vollumfänglich geniessen, weil das Angebot nicht angemessen ist und für bestimmte Personengruppen ein Hindernis darstellt. Dank geeigneter Massnahmen wie Untertitelung oder Verdolmetschung in Gebärdensprache sowie Vorlesen schriftlicher Informationen am Bildschirm und Filmen mit Audiodeskription können diese Hindernisse überwunden werden. Dieser Perspektivenwechsel wurde in jüngster Zeit auch im Schweizer Recht verankert. Er wurde in der Botschaft des Bundesrates zum Behindertengleichstellungsgesetz sehr zutreffend beschrieben: "Als systemische Hindernisse erweisen sich architektonische Barrieren, fehlende behindertengerechte hygienische Einrichtungen, mangelnde Berücksichtigung spezieller Bedürfnisse von Menschen mit Wahrnehmungsschwierigkeiten in den Medien und in öffentlichen Verkehrsmitteln, ungenügende Teilzeitbeschäftigungsmöglichkeiten usw. Soziale Strukturen tragen dazu bei, die Behinderung zu schaffen oder zu verstärken.» [2]

Verstärkt laufen Anstrengungen seitens der Lösungsanbieter, ihre Dienstleistungen behindertengerecht $\mathrm{zu}$ entwickeln und anzubieten. Am 5. Schweizerischen E-Healthcare-Kongress, welcher ja neu im Forschungszentrum des Schweizer Paraplegiker-Zentrums in Nottwil stattfindet, soll dieses Thema in einem Symposium erstmals thematisiert werden. Die Schweiz steht am Anfang dieses grossen und wichtigen Themas. Anhand zweier Praxisbeispiele sei auf erfolgreiche Entwicklungen hingewiesen. 


\section{Webportal MyHandicap.com}

MyHandicap.com ist ein Webportal für Menschen mit Behinderungen. Getragen wird es durch die gleichnamige Stiftung. Das Ziel von Stiftung und Portal ist die Förderung von Menschen mit einer Behinderung, insbesondere deren umfassende gesellschaftliche Integration, die Erleichterung des Alltages der Betroffenen und der Informationsaustausch von Behinderten untereinander und von Behinderten mit Nichtbehinderten.

Ein Internetportal für behinderte Menschen soll zielgruppenrelevante Themen bündeln und den Betroffenen aus einer Quelle zur Verfügung stellen. Die Informationsplattform MyHandicap.com möchte in Zukunft den zentralen Anlaufpunkt für alle Themen im Zusammenhang mit Behinderungen darstellen. Ein Internetportal ist eine ideale Informations- und Kommunikationsplattform für Menschen mit Mobilitätseinschränkungen. Mit zielgruppenspezifischen Angeboten und Inhalten wird das Portal MyHandicap.com eine neue Brücke in die reale Welt schaffen und so zur Reintegration und Verbesserung des Lebens körperbehinderter Menschen beitragen. MyHandicap.com versteht sich als Mittler und soll zu einem länderübergreifenden Netzwerk ausgebaut werden. Der Aufbau des Internetportals wird unter Einbezug der WAI-Richtlinien erfolgen und behindertengerecht realisiert.

\section{Qualilife - Zugangstechnologien für behinderte und ältere Menschen}

Spitalzimmer sind bereits mit verschiedenen Technologien für Patienten ausgerüstet: Telefon, Umfeldkontrolle (Basis), Alarme, Fernsehen und Radio. Grundsätzlich sind diese Technologien für Patienten nur zugänglich, wenn diese einen Knopf drücken, eine Fernbedienung bedienen oder sogar aufstehen und den Lichtschalter anschalten können. Da behinderte Patienten (permanent oder temporär) diese Fähigkeiten nicht besitzen, ist dies in vielen Fällen ein Problem. Dadurch werden natürlich die zur Verfügung stehenden Technologien nutzlos, was wiederum den Zeitaufwand des Spitalpersonals erhöht.

Gleichzeitig fühlen sich Patienten oft isoliert und wurden aus dem alltäglichen Leben gerissen. Zum Beispiel ein Internetzugang und die Möglichkeit, elektronische Nachrichten an das Spitalpersonal zu senden, gibt Patienten «mehr Kontrolle über ihr Schicksal» und eine bestmögliche Selbständigkeit zurück. In einem Spital kommen Bedürfnisse von vielen verschiedenen
Ausgangslagen zusammen. Deshalb braucht es eine Lösung, die einfach in der Handhabung, aber trotzdem für alle Patienten komplett zugänglich ist, ungeachtet des Alters oder der körperlichen Verfassung. Das heisst, die gleiche Einrichtung muss für Patienten ohne körperliche Schwächen sowie für komplett gelähmte oder bewegungsunfähige Menschen benutzbar sein.

Ein neues System muss die bis jetzt vorhandenen Technologien, wie Telefon, Umfeldkontrolle, Alarme usw., kontrollieren können, sich aber vor dem Fortschritt nicht verschliessen und auch die neuen Kommunikationsformen E-Mail, Internet, Fax, SMS usw. beinhalten. Spitalpatienten verbringen viele Stunden täglich im Bett, und deshalb gehört natürlich auch die Unterhaltungselektronik zu einem wichtigen Angebot (TV, DVD, Musik und Spiele). In der heutigen Zeit sind Neuerungen an der Tagesordnung. Aus diesem Grund kann nur ein System überleben, das sehr flexibel ist und eine komplette Kompatibilität auch mit zukünftigen Technologien oder Standards garantiert, ohne dass dabei die ganze Installation ersetzt werden muss.

Die Softwareplattform QualiWORLD (www. qualilife.com) ermöglicht all dies. Installiert auf einem Pentium-III-Prozessor mit Microsoft Windows XP Pro oder auf dem neuen Microsoft Windows Media Center Edition 2005, heisst es: eine Lösung für jeden Patienten und für jedes Bedürfnis. QualiWORLD wurde entwickelt, um Menschen mit körperlichen Behinderungen jeden Alters Zugriff auf die gängigsten und wichtigsten Technologien zu gewähren. Im Detail bedeutet dies: telefonieren und sprechen (verbale Kommunikation), E-Mail, SMS und Fax versenden, Texte verfassen (schriftliche Kommunikation), im Internet navigieren, Fernsehen, Radio hören, DVDs ansehen, Panoramen in der virtuellen Realität geniessen, Karten- und Schachspielen, IP-Kameras kontrollieren (Unterhaltung und Multimedia). Mittels Infrarot, Powerline (PLC), EiB, Bus, Radiofrequenzen usw. können alle elektronischen Geräte im Umfeld kontrolliert werden. Alarme können über Telefon, SMS, Alarmsysteme usw. ebenfalls kontrolliert werden.

All diese Funktionen sind zugänglich über verschiedene bereits integrierte Mausersatzlösungen und eine komplett konfigurierbare virtuelle Tastatur. QualiWORLD ist auch kompatibel mit allen existierenden assistiven Technologien wie Augensteuerungssystemen, Spracherkennungssystemen, Touchscreens, Joysticks, Taster usw.

Mit dieser Lösung und mit der somit neugewonnenen Selbständigkeit wird die Patientenzufriedenheit nachweislich erhöht, zusätzliches 
Einkommen generiert und Pflegepersonal von Anfragen ohne medizinischen Charakter entbunden. Stumme Menschen oder Patienten mit Sprachschwierigkeiten können frei und klar ihre Bedürfnisse und Anliegen zum Ausdruck bringen. Frustrationsraten werden gesenkt und die Arbeit des Pflegepersonals und der Ärzte erleichtert.

Die Clinique Romande de Réadaptation der SuvaCare aus Sion hat QualiWORLD auf drei ver- schiedene Arten eingesetzt. Zum einen in der Abteilung Ergotherapie, wo die Therapeuten mit den Patienten arbeiten, die besten Zugangslösungen aussuchen und natürlich Fortschritte feststellen können. Zum anderen als Grossbildschirmlösung. Hier wurde QualiWORLD auf das Microsoft Media Center Edition mit einem 32"-Bildschirm installiert und kann in jedes Zimmer des Spitals gebracht und sofort angewendet werden.

\section{eHealthCare.ch \\ ICT und neue Technologien in Medizin und Gesundheitswesen}

Konferenz am 5. Schweizerischen E-Healthcare-Kongress

41 PRACTICE Accessibility - Zugang für Behinderte zu moderner Technologie

25. Oktober, 11.00-12.30 Uhr 